\title{
PSORIASIS- A CLINICAL STUDY IN THANJAVUR MEDICAL COLLEGE
}

\author{
Ramasamy Sindhuja1, Mariaviagulam Vinnarasan², Gopalan Balaji3, Ponnusamy Pavithra ${ }^{4}$, Ganapathy Senthil5, \\ Balachandran Sarath Chandran ${ }^{6}$
}

${ }_{1}^{1}$ Associate Professor, Department of Dermatology, Thanjavur Medical College.

${ }^{2}$ Senior Assistant Professor, Department of Dermatology, Thanjavur Medical College.

${ }^{3}$ Senior Assistant Professor, Department of Dermatology, Chengalpet Medical College.

4Junior Resident, Department of Dermatology, Thanjavur Medical College.

${ }_{5}^{5}$ Senior Assistant Professor, Department of Dermatology, Thanjavur Medical College.

6Junior Resident, Department of Dermatology, Chengalpet Medical College.

\section{BACKGROUND}

\section{ABSTRACT}

Psoriasis is a common papulosquamous relapsing autoimmune skin disease with genetic predisposition and environmental factors being the triggers. The knowledge of the prevalence of common dermatoses will be useful for adequate treatment and care of the patients.

The aim of the study is to determine the pattern and prevalence of psoriasis in our medical college.

\section{MATERIALS AND METHODS}

This study is a hospital-based descriptive study that includes 200 self-reporting patients of age group 4 - 80 years with psoriasis who attended the skin OP for 6 months from October 2016 to March 2017 in our medical college situated in South India.

\section{RESULTS}

Chronic plaque psoriasis is the most common (52.8\%) clinical pattern observed irrespective of age and sex. Nail involvement was common. Comorbidities associated were diabetes mellitus, hypertension, hypothyroidism, myocardial infarction, alcoholism, peripheral neuropathy and depression.

\section{CONCLUSION}

Data correlates with previous hospital-based prevalence studies of psoriasis.

\section{KEYWORDS}

Psoriasis, Peripheral Neuropathy.

HOW TO CITE THIS ARTICLE: Sindhuja R, Vinnarasan M, Balaji G, et al. Psoriasis- a clinical study in Thanjavur medical college. J. Evolution Med. Dent. Sci. 2017;6(53):4034-4038, DOI: 10.14260/Jemds/2017/872

\section{BACKGROUND}

Psoriasis is a common, non-communicable, chronic relapsing autoimmune skin disease. It is a lifelong disease associated with a number of morbidities and has an impact on the psychosocial aspects. There are a growing number of population-based studies providing worldwide prevalence estimates of psoriasis. Prevalence of psoriasis varies in different parts of the world. According to published reports, prevalence in different populations varies from $0 \%$ to $11.8 \%{ }^{[1-4]}$ The exact prevalence of psoriasis in India is not known and we rely on Western data. The information on prevalence would be useful for planning strategies to manage these diseases. This point prevalence study was undertaken to determine the epidemiological pattern of psoriasis. In the above situation, this hospital-based study was conducted.

\section{MATERIALS AND METHODS}

The study was a descriptive study on 200 self-reported patients who attended Dermatology Outpatient Department

Financial or Other, Competing Interest: None.

Submission 03-05-2017, Peer Review 22-06-2017,

Acceptance 27-06-2017, Published 03-07-2017.

Corresponding Author:

Dr. Ramasamy Sindhuja,

Associate Professor,

Department of Dermatology,

Thanjavur Medical College.

E-mail:dhunsi75@gmail.com

DOI: $10.14260 /$ jemds $/ 2017 / 872$ at Thanjavur Medical College situated in southern part of India. It was conducted during a period of 6 months from October 1, 2016 to March 31, 2017. We enrolled all patients with no exclusion based on sex, nationality or socioeconomic status. Details like age, sex, age of onset of lesions, duration and pattern of lesions with associated diseases, provocative factors, blood investigations like complete blood count, blood sugar levels, thyroid function test and urine analysis were done for all patients. All the patients were subjected to nerve conduction study to rule out peripheral neuropathy. Clinical diagnosis of psoriasis was made by detailed history, physical examination and biopsy if needed. The diagnosis of psoriasis is made based on clinical evaluation and physical examination. Data were analysed using descriptive analyses for co-morbidities, types of psoriasis, nail and joint involvement, age of onset, provocative factors and associated skin conditions.

\section{RESULTS}

Proportion of Psoriasis Cases among Total Cases Attending OPD

Among the total number of patients attended during this period in the Dermatology Outpatient Department of Thanjavur Medical College, about 200 patients were diagnosed to have psoriasis. It comes to about $1.3 \%$ of total number $(15,385)$ of new cases. 


\section{Sex Ratio}

Of the total 200 patients with psoriasis 143 were males and 67 were females. (Male: Female ratio was 2.5: 1).

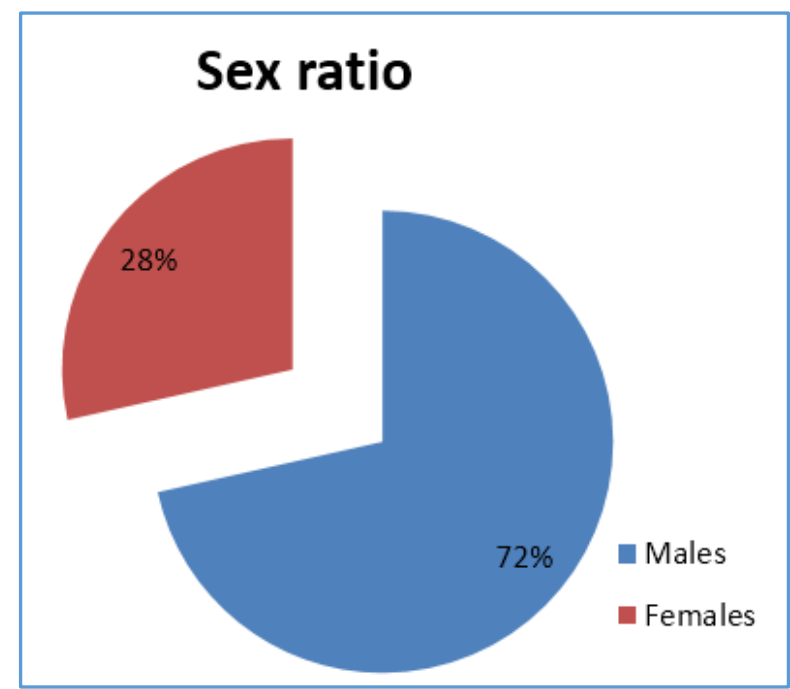

\section{Clinical Pattern}

Chronic plaque psoriasis [Figure 1] was the most common type. It involved about 106 patients, i.e. about $52.8 \%$ of the total patients. The second most common was palmoplantar [Figure 2 and 3], which constitutes about 73 patients i.e. $36.4 \%$, then scalp about $6.4 \%$ [Figure 4]. The remaining flexural, erythrodermic, guttate and pustular constitute less than $5 \%$. Only single case of linear presentation was there [Figure 5].

\section{Age of Onset}

The most common age of onset is between 20 to 30 years, which is about $32 \%$ (64 cases) of the total cases which is in concordance with any other study. 48 cases are between 30 to 40 years of age (24\%). $18 \%$ patients (36 cases) are above 40 to 50 years of age. $16 \%$ patients (32 cases) are above 50 years. Only $10 \%$ of patients (20cases) are under 20 years of age in our study.

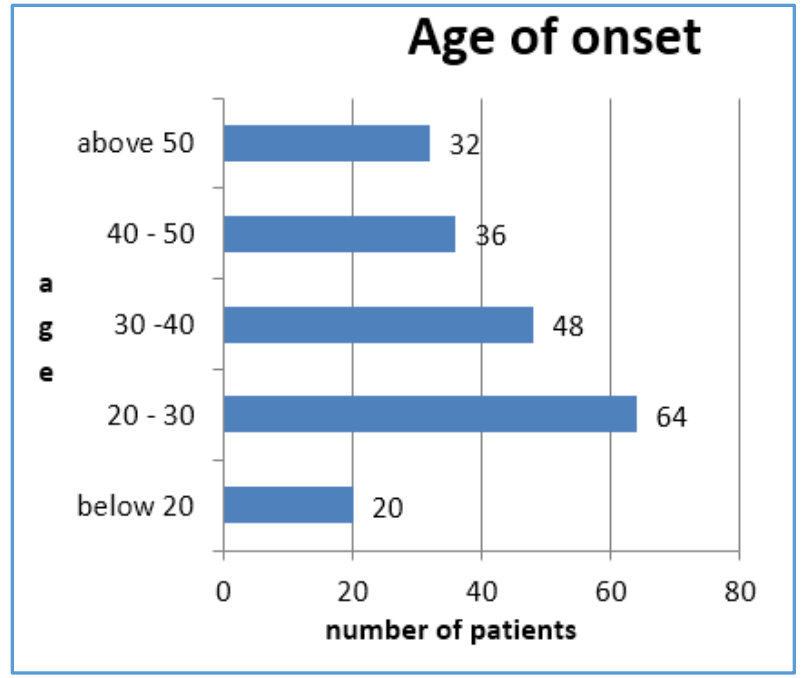

\section{Nail Involvement}

Finger nails were commonly involved than toe nails and $45 \%$ of the patients had nail involvement, which is in concordance with study conducted by Lewis et al.[5] The most common changes were pitting [Figure 6], which accounts for $70 \%$ of the nail involvement and subungual hyperkeratosis [Figure 7 ] accounts for $50 \%$. Both nail pitting and subungual hyperkeratosis were seen in $25 \%$ of the patients. Other nail changes like oil drop sign, thickening of nail plate and onycholysis are seen in less than $5 \%$ of the patients. Nail involvement was seen in almost all psoriatic arthritis patients.

\section{Joint Involvement}

Joint involvement was seen in $5 \%$ of our psoriatic patients (10 cases), which is in concordance with the study conducted by Baker et al.[6] The most common joint involvement was oligoarthritis involving proximal interphalangeal joint (4 cases), knee joint involvement was seen in 3 cases, distal interphalangeal involvement was seen in 2 cases and 1 patient had rheumatoid-like joint involvement.

All the cases were seronegative patients.

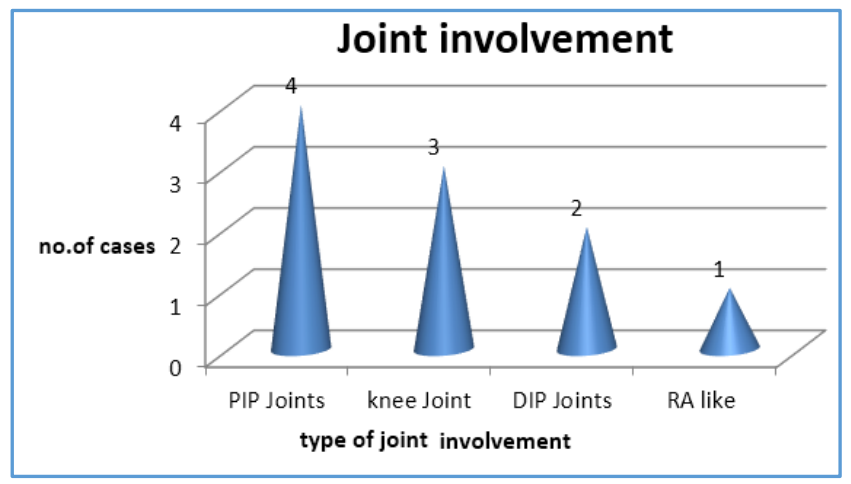

\section{Comorbidities}

30 patients had diabetes mellitus, 22 patients were hypertensive, 15 patients hypothyroid and 10 patients had coronary artery disease. About 50 patients were obese, 20 patients had habit of alcohol intake, 5 patients had peripheral neuropathy and 5 were taking treatment for depression.

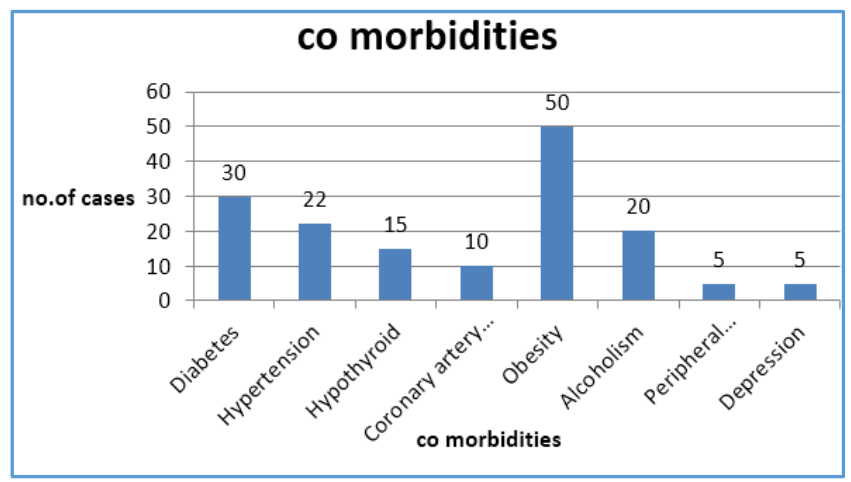

\section{Associated Skin Conditions}

Autoimmune skin conditions like vitiligo, lichen planus and alopecia areata were seen in 11, 9 and 8 patients respectively. Lower limb cellulitis was seen in 14 patients with psoriasis. Infective skin diseases like wart was seen in 7 patients and 
herpes zoster had occurred in 6 patients with psoriasis; 2 psoriatic patients had bullous pemphigoid.

\section{Provocative Factors}

18 patients had history of drug intake before the onset of psoriasis ( 5 had ACE inhibitors, 6 had native treatment, 4 had NSAIDS and other 3 had antimalarials); 5 patients had fever and infection, 4 patients had history of trauma previous to the onset of psoriasis. Sunlight stimulated psoriasis in patients and pregnancy in 2 patients.

\section{Provocative Factors}

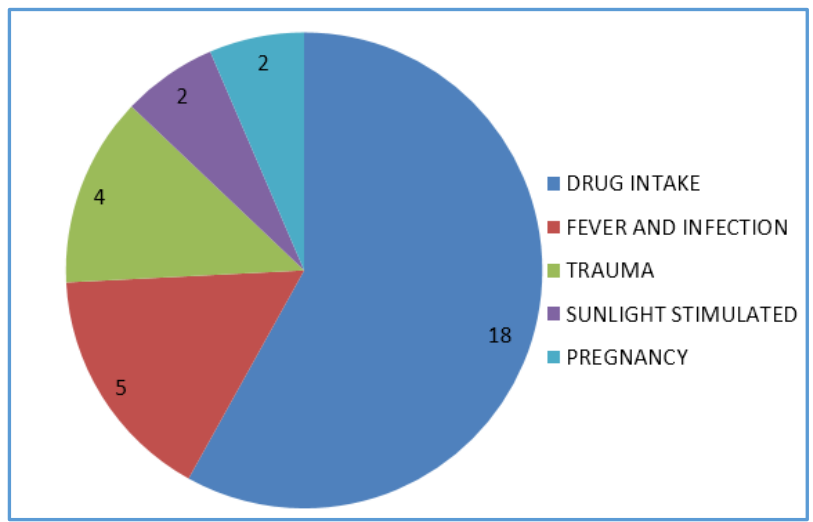

\section{RESULTS}

From the available studies, the prevalence of psoriasis in India ranges from 0.44 to $2.8 \% .{ }^{[8]}$ The proportion of psoriasis out of all the skin diseases in our study was $1.3 \%$. We report a male predominance of psoriasis similar to other reports. Chronic plaque type psoriasis was the most common type of psoriasis in our study.

Nail involvement is common in psoriasis and can be the initial and the only site of involvement in some patients. Morphology of nail changes depends on whether nail matrix, nail bed or hyponychium has been affected. Nail involvement is more common in those who have concurrent psoriatic arthritis. Our study showed nail changes in $45 \%$, which is in concurrent with the study by Bedi et al. Nail involvement is most commonly associated with joint involvement.

The most common joint involvement was oligoarthritis involving proximal interphalangeal joint with enthesitis. Rheumatoid factor was negative for all patients with joint involvement. Skin lesions preceded arthritis in all our psoriatic patients.

Our patients have multiple co-morbidities like hypertension, diabetes, ischaemic heart disease, obesity, peripheral neuropathy and hypothyroidism. Psoriasis increases the risk of obesity. A study from UK showed higher adjusted odds of obesity in patients with severe psoriasis $(\mathrm{OR}=1.8)$ than in patients with mild psoriasis $(\mathrm{OR}=1.3)$ compared with patients without psoriasis. Vice versa, obesity is linked to psoriasis due to its chronic proinflammatory state as postulated by Basko-Plluska JL et al.[9]

Other autoimmune diseases like vitiligo, lichen planus, bullous pemphigoid and alopecia areata were associated in our psoriatic patients. These autoimmune diseases were identified during the course of the disease. These diseases did not interfere with the course of the psoriasis. Infective diseases like wart cellulitis and herpes zoster occurred during the course of psoriasis and these diseases resulted in mild exacerbation of psoriasis. Native medicine, depot steroids and antihypertensive drugs were the main provocative factors for psoriasis in our study.

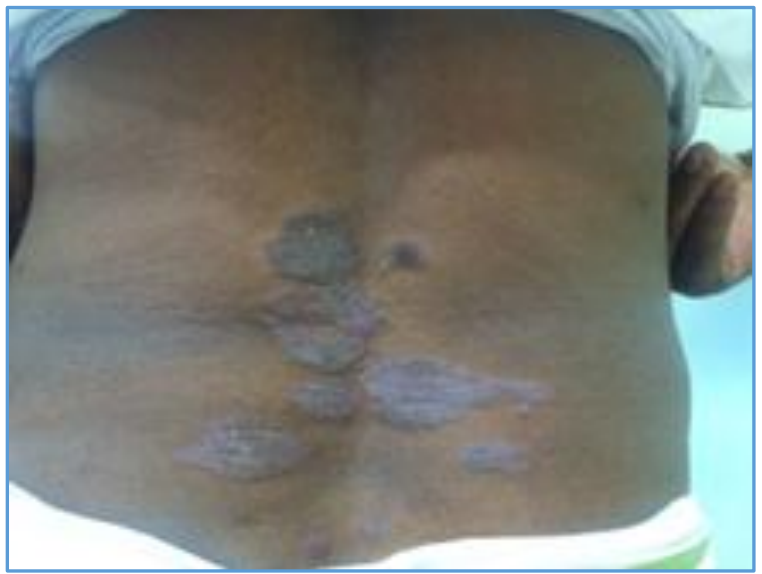

Figure 1. Chronic Plaque Psoriasis

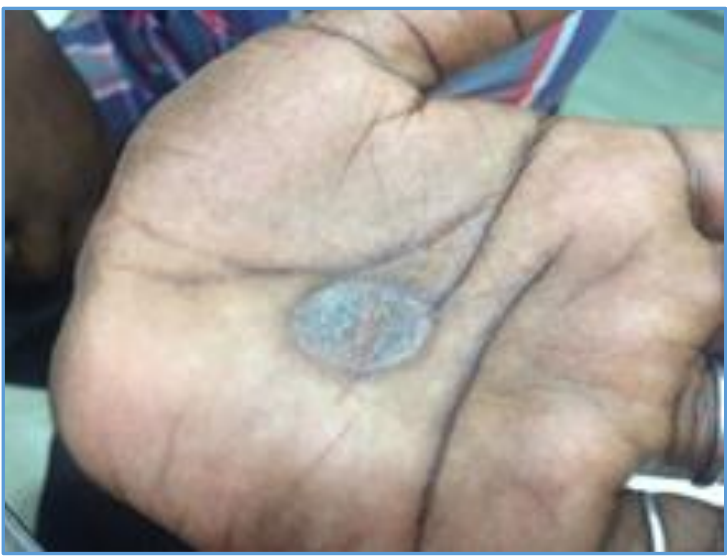

Figure 2. Palmar Psoriasis

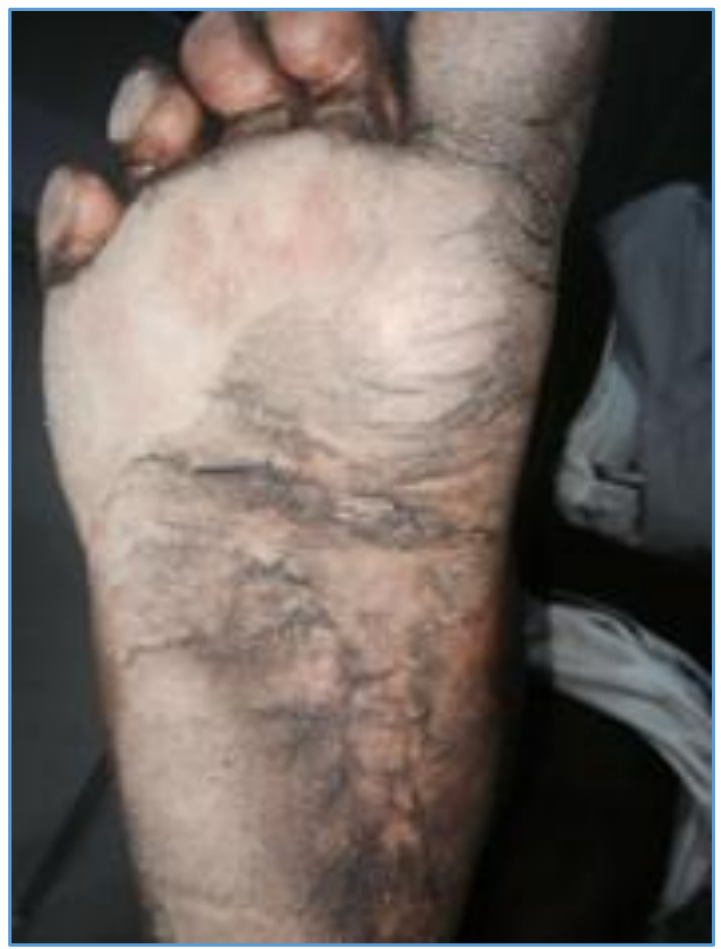

Figure 3. Plantar Psoriasis 


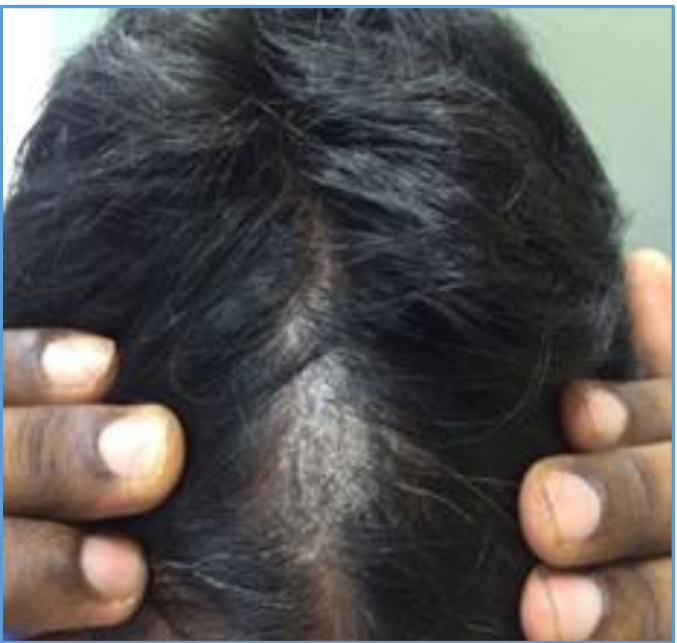

Figure 4. Scalp Psoriasis

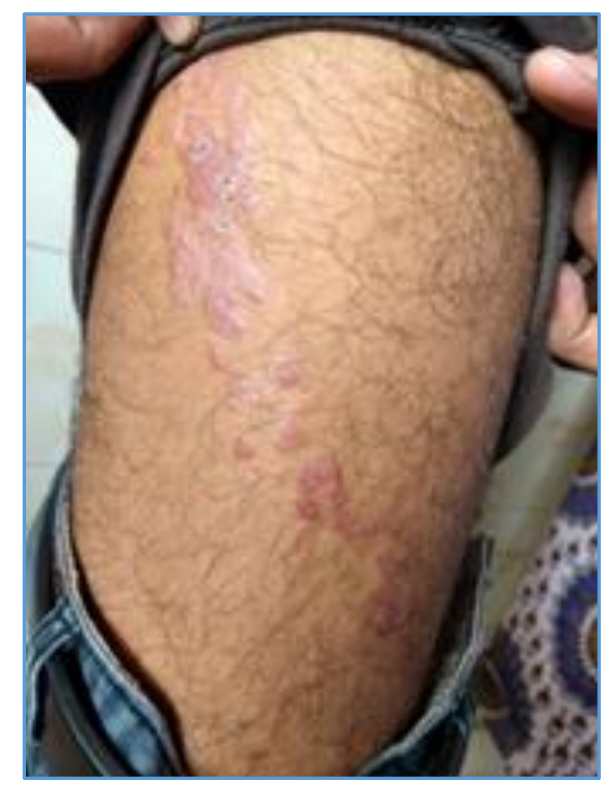

Figure 5. Linear Psoriasis

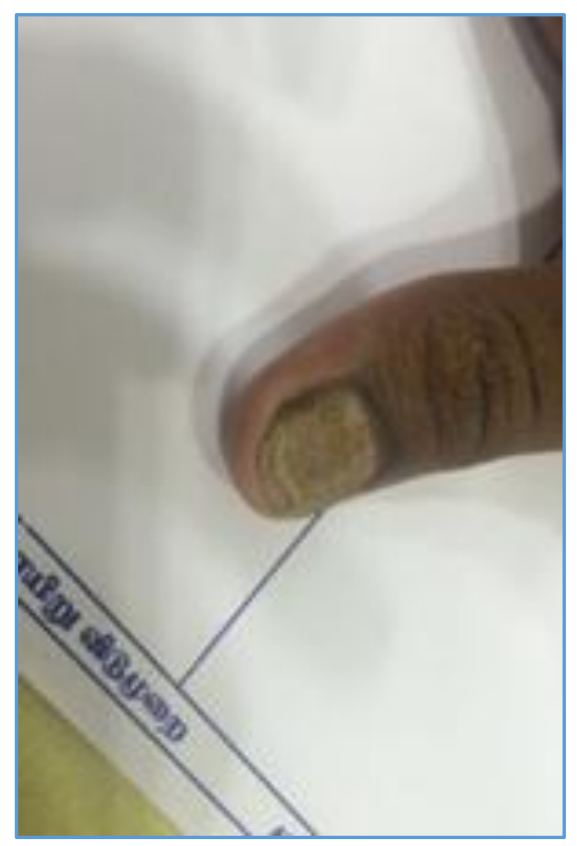

Figure 6. Nail Psoriasis

\section{DISCUSSION}

Psoriasis is regarded as an autoimmune papulosquamous disease, in which genetic and environmental factors have a significant role. The name of the disease is derived from Greek word "psora," which means "itch." The skin cells mature and are shed from the skin's surface every 28 to 30 days.[6] When psoriasis develops, the skin cells mature in 3 to 6 days and move to skin surface. Instead of being shed, the skin cells pile up causing the visible lesions. It is also found that genes that cause psoriasis can determine how a person's immune system reacts. These genes can cause psoriasis or other immune-mediated conditions such as rheumatoid arthritis and type 1 diabetes. ${ }^{[7]}$

The characteristic lesion of psoriasis is well-defined erythematous plaques with silvery white scales mainly involving lumbosacral area, bony prominences and extensor surface of extremities, which exhibits Koebner phenomenon (i.e. occurrence of isomorphic lesions along the line of trauma). Koebner phenomenon indicates disease activity. The removal of psoriatic scales (candle wax sign) usually reveals an underlying smooth, glossy membrane (Berkeley membrane) with small bleeding points where the thin suprapapillary epithelium is torn off (Auspitz sign).

Clinical variants of psoriasis includes chronic stable plaque psoriasis, guttate psoriasis, pustular psoriasis, rupioid psoriasis, elephantine psoriasis, ostraceous psoriasis, unstable psoriasis, psoriatic arthritis and erythrodermic psoriasis. Based on site psoriasis was classified into scalp psoriasis, nail psoriasis, palmoplantar psoriasis and inverse psoriasis. Guttate psoriasis mainly occur in children and young adult usually following group A beta-haemolytic streptococcal infection, the lesions are small ranging from 0.5 to $1 \mathrm{~cm}$ in diameter, round or oval, mainly involving trunk and proximal part of limbs. Pustular psoriasis is classified into localised and generalised. Localised pustular psoriasisFurther psoriasis is classified into palmoplantar pustulosis, and Acrodermatitis Continua of Hallopeau. Generalised pustular psoriasis is classified into acute GPP, pustular psoriasis of pregnancy, infantile and juvenile type, and circinate type.

Psoriatic arthritis is an inflammatory arthritis associated with skin and/or nail psoriasis with negative serological test for rheumatoid factor and the absence of rheumatoid nodules. Moll and Wright Classified Psoriatic Arthritis into peripheral mono or oligoarthritis is the most common form, distal interphalangeal arthritis, symmetrical rheumatoid-like polyarthritis, arthritis mutilans and axial arthritis.

Mucosal involvement by psoriasis appears to be rare, but has been associated with cutaneous involvement by pustular and erythrodermic type. Geographic tongue is commonly associated with generalised type of pustular psoriasis.

\section{CONCLUSION}

Our cohort of patients showed a similar clinical profile and outcome as our Indian psoriasis population. More research and detailed prospective studies need to be done to delineate the natural course of the disease, which varies in different individuals and also according to the clinical pattern of the disease. Prevention of psoriasis has barely been studied. A prerequisite would be that risk factors are identified in a consistent and reliable way. Also there is need for a more effective targeted therapy for a better outcome. 


\section{REFERENCES}

[1] Kaur I, Kumar B, Sharma VK, et al. Epidemiology of psoriasis in a clinic from North India. Indian J Dermatol Venereol Leprol 1986;52(4):208-12.

[2] Bedi TR. Psoriasis in North India. Geographical variations. Dermatologica 1977;155(5):310-4.

[3] Swanbeck G, Inerot A, Martinsson T, et al. A population genetic study of psoriasis. $\mathrm{Br} \mathrm{J}$ Dermatol 1994;131(1):32-9.

[4] Farber EM, Nall L. Epidemiology: natural history and genetics. In: Roenigk HH, Maibach HI, (eds). Psoriasis. New York: Dekker 1998:107-57.

[5] Lewin K, DeWit S, Ferrington RA. Pathology of the fingernail in psoriasis. A clinicopathological study. Br J dermatol 1972;86(6):555-63.
[6] Baker H. Epidermiological aspects of psoriasis and arthritis. Br J Dermatol 1966;78(5):249-61.

[7] Gottlieb SL, Gilleaudeau P, Johnson R, et al. Response of psoriasis to a lymphocyte-selective toxin (DAB389IL-2) suggests a primary immune, but not keratinocyte, pathogenic basis. Nat Med 1995;1(5):442-7.

[8] Raychaudhuri SP, Rein G, Farber EM. Neuropathogenesis and neuropharmacology of psoriasis. In Int J Dermato 1995;34(10):685-93.

[9] Basko-Plluska JL, Petronic-Rosic V. Psoriasis: epidemiology, natural history, and differential diagnosis. Psoriasis: Targets and Therapy 2012;2: 67-7. 